\title{
Mortality predictors in critically ill patients with acute kidney injury requiring continuous renal replacement therapy
}

\author{
Kristianne Rachel P. Medina-Liabres ${ }^{1}$, Jong Cheol Jeong ${ }^{1}$, Hyung Jung $\mathrm{Oh}^{2,3}$, Jung Nam $\mathrm{An}^{4}$, Jung Pyo Lee ${ }^{5,6}$, \\ Dong Ki Kim ${ }^{6}$, Dong-Ryeol Ryu ${ }^{7,8}$, Sejoong $\mathrm{Kim}^{6}$ \\ ${ }^{1}$ Department of Internal Medicine, Seoul National University Bundang Hospital, Seongnam, Republic of Korea \\ ${ }^{2}$ Ewha Institute of Convergence Medicine, Ewha Womans University Mokdong Hospital, Seoul, Republic of Korea \\ ${ }^{3}$ Research Institute for Human Health Information, Ewha Womans University Mokdong Hospital, Seoul, Republic of Korea \\ ${ }^{4}$ Department of Internal Medicine, Hallym University Sacred Heart Hospital, Anyang, Republic of Korea \\ ${ }^{5}$ Department of Internal Medicine, Seoul National University Boramae Medical Center, Seoul, Republic of Korea \\ ${ }^{6}$ Department of Internal Medicine, Seoul National University College of Medicine, Seoul, Republic of Korea \\ ${ }^{7}$ Department of Internal Medicine, Ewha Womans University, Seoul, Republic of Korea \\ ${ }^{8}$ Tissue Injury Defense Research Center, Ewha Womans University, Seoul, Republic of Korea
}

Background: Because of high cost of continuous renal replacement therapy (CRRT) and the high mortality rate among severe acute kidney injury patients, careful identification of patients who will benefit from CRRT is warranted. This study determined factors associated with mortality among critically ill patients requiring CRRT.

Methods: This was a retrospective observational study of 414 patients admitted to the intensive care unit of four hospitals in South Korea who received CRRT from June 2017 to September 2018. Patients were divided according to degree of fluid overload (FO) and disease severity. The Cox proportional hazards model was used to explore the effect of relevant variables on mortality.

Results: In-hospital mortality rate was $57.2 \%$. Ninety-day mortality rate was $58.5 \%$. Lower creatinine and blood pH were significant predictors of mortality. A one-unit increase in the Sequential Organ Failure Assessment (SOFA) score was associated with increased risk of and 90-day mortality (hazard ratio [HR], 1.07; $p<0.001$ ). The risk of 90-day mortality in FO patients was $57.2 \%(p<0.001$ ) higher than in those without FO. High SOFA score was associated with increased risk for 90-day mortality (HR, 1.79; $p=0.03$ and HR, 3.05; $p=0.001$ ) in patients without FO and with FO $\leq 10 \%$, respectively. The highest mortality rates were in patients with FO $>10 \%$, independent of disease severity.

Conclusion: FO increases the risk of mortality independent of other factors, including severity of acute illness. Prevention of FO should be a priority, especially when managing the critically ill.

Keywords: Acute kidney injury, Continuous renal replacement therapy, Critical illness, Mortality

Received: October 28, 2020; Revised: February 19, 2021; Accepted: March 7, 2021

Editor: Seung-Yeup Han, Keimyung University, Daegu, Republic of Korea

Correspondence: Sejoong Kim

Department of Internal Medicine, Seoul National University Bundang Hospital, 82 Gumi-ro 173beon-gil, Bundang-gu, Seongnam 13620, Republic of Korea. E-mail: sejoong@snubh.org

ORCID: https://orcid.org/0000-0002-7238-9962

Copyright (C) 2021 by The Korean Society of Nephrology

(a) This is an Open Access article distributed under the terms of the Creative Commons Attribution Non-Commercial and No Derivatives License (http:// creativecommons.org/licenses/by-nc-nd/4.0/) which permits unrestricted non-commercial use, distribution of the material without any modifications, and reproduction in any medium, provided the original works properly cited. 


\section{Introduction}

Acute kidney injury (AKI) is a common complication among critically ill patients worldwide and is associated with substantial morbidity and mortality rates of $50 \%$ to $90 \%$ [1]. Approximately $5 \%$ to $10 \%$ of AKI patients require acute renal replacement therapy (RRT) during intensive care unit (ICU) admission [2-4]. AKI patients requiring RRT are reported to have very high mortality rates, as much as $50 \%$ to $80 \%$ [5]. Risk factors for mortality include advanced age, sepsis, disease severity and number of failing organs, need for mechanical ventilation, presence of circulatory shock, and oliguria [6]. Several studies have also established the relationship between fluid overload (FO) and mortality [7-10].

Continuous RRT (CRRT) is the preferred renal replacement modality in the management of critically ill patients with hemodynamic instability and AKI [5]. The Kidney Disease: Improving Global Outcome (KDIGO) Clinical Practice Guideline for AKI suggests that CRRT be used for hemodynamically unstable patients and patients with acute brain injury or other causes of increased intracranial pressure [11]. Many studies show that CRRT offers superior hemodynamic stability, metabolic clearance, and volume control. Other advantages of CRRT include enhanced clearance of inflammatory mediators and better preservation of cerebral perfusion among patients with acute brain injury or fulminant hepatic failure [12].

The limited availability in some areas and the high cost of CRRT, in addition to the high mortality rate among critically ill patients with severe AKI, warrant careful selection of patients who will benefit from it. Identifying patients who are most likely to have positive outcomes with CRRT is challenging. More studies are needed to identify such patients to guide therapeutic decisions, optimize limited resources, and provide realistic prognostic information to patients and their families. This study aims to determine mortality rates and identify factors associated with mortality among critically ill patients in the ICU with AKI who received CRRT.

\section{Methods}

\section{Research design}

This is multicenter observational study comprising all AKI patients who were admitted and received CRRT in the ICUs of Seoul National University Bundang Hospital, Seoul National University Hospital, Seoul National University Boramae Medical Center, and Ehwa Womans University Mokdong Hospital, from June 2017 to September 2018.

This study was approved by the Institutional Review Boards of Seoul National University Bundang Hospital (No. 1801-44-106), Seoul National University Hospital (No. 1801036-913), Seoul National University Boramae Medical Center (No. 10-2018-05), and Ehwa Womans University Mokdong Hospital (No. 2018-01-071). This study was performed in accordance with the Declaration of Helsinki. Informed consent was waived because the study is retrospective and noninterventional in nature.

\section{Inclusion and exclusion criteria}

All patients with AKI who received CRRT in the ICUs of Seoul National University Bundang Hospital, Seoul National University Hospital, Seoul National University Boramae Medical Center, and Ehwa Womans University Mokdong Hospital from June 2017 to September 2018 were eligible. Patients already on chronic dialysis before the study period were excluded.

\section{Data collection}

Records of ICU patients with AKI who received CRRT over the study period were retrieved. Baseline demographics such as age and sex, preexisting comorbid conditions, and etiology of AKI were collected. Although AKI is often multifactorial, we classified patients into one of four etiologic groups: (1) septic, (2) cardiogenic, (3) postoperative, and (4) others. Use of mechanical ventilation and vasopressor(s), urine output 24 hours prior to CRRT, length of stay in the ICU and in the hospital, and time on CRRT were also noted. Disease severity was assessed using the Sequential Organ Failure Assessment (SOFA) score. Laboratory findings were also recorded.

\section{Definitions}

The AKI diagnostic criteria utilized in this study were in accordance with the 2012 KDIGO Clinical Practice Guideline for AKI: increase in serum creatinine $(\mathrm{SCr}) \geq 0.3 \mathrm{mg} / \mathrm{dL}$ within 48 hours, increase in $\mathrm{SCr} \geq 1.5$ times the baseline, or 
urine volume $<0.5 \mathrm{~mL} / \mathrm{kg} /$ hour for 6 hours [11]. The degree of FO was expressed as percent FO, which was calculated as follows: [(weight at start of CRRT - baseline body weight)/ baseline body weight] $\times 100$.

\section{Groups}

Patients were grouped according to presence and degree of FO: group 1, no FO; group 2, FO $\leq 10 \%$; and group 3 , FO > $10 \%$. Patients were also classified according to SOFA score: A, low SOFA score $(<10)$ and B, high SOFA score $(\geq 10)$, using the median SOFA score.

\section{Outcomes}

The primary outcomes were in-hospital mortality and 90day mortality. We analyzed 90-day mortality to reduce bias related to short-term prognosis brought about by the acute illness.

\section{Statistical analysis}

Categorical data are expressed as the number of cases and percentages. Continuous data are expressed as the mean \pm standard deviation (SD). Statistical comparisons were made between survivors and non-survivors using the independent t-test and the chi-square test, as appropriate. The Cox proportional hazards model was used to explore the effect of variables on mortality. The adjusted model included age, sex, hypertension, diabetes mellitus (DM), malignancy, and sepsis. Mortality data were analyzed using Kaplan-Meier survival curves. Statistical significance was defined as a $\mathrm{p}$-value of $<0.05$. All statistical analyses were performed using IBM SPSS version 20 (IBM Corp., Armonk, NY, USA).

\section{Results}

\section{Baseline characteristics}

We identified 414 critically ill patients with AKI who received CRRT. When patients were divided into survivor and non-survivor groups, there was no difference in the age or sex distribution ( $\mathrm{p}=0.50$ and $\mathrm{p}=0.90$, respectively). The mean age was $65.8 \pm 15.3$ years in the survivor group and $66.8 \pm 14.4$ years in the non-survivor group. A majority of the patients in both groups ( $67.8 \%$ and $68.4 \%$, respectively) were male. The two most common comorbidities among all patients were hypertension and DM. The non-survivors had a higher mean SOFA score ( $10.3 \pm 3.8$ vs. $8.9 \pm 3.2, \mathrm{p}<0.001)$, lower mean arterial pressure $(77.8 \pm 15.8 \mathrm{mmHg}$ vs. $83.2 \pm$ $17.6 \mathrm{mmHg}, \mathrm{p}=0.001$ ), and required at least one vasopressor $(78.1 \%$ vs. $62.1 \%, \mathrm{p}<0.001)$. A majority of the patients in both groups required mechanical ventilation (56.5\% among survivors vs. $74.3 \%$ among non-survivors, $\mathrm{p}<0.001)$. Sepsis was the most common cause of AKI in either group. The mean SCr was lower in the non-survivor group $(2.9 \pm 1.7$ $\mathrm{mg} / \mathrm{dL}$ vs. $4.3 \pm 3.1 \mathrm{mg} / \mathrm{dL}$ in the survivor group, $\mathrm{p}<0.001)$. A higher proportion of patients in the non-survivor group had FO at the time of CRRT initiation ( $51.1 \%$ vs. $34.5 \%, \mathrm{p}=0.001$ ). Table 1 shows the baseline clinical characteristics of the patients, stratified by in-hospital mortality.

\section{Outcomes}

The in-hospital mortality rate was $57.2 \%$ (237 of 414). Of the 177 patients who survived to hospital discharge, five patients expired in the 90 days following the start of CRRT. The 90day mortality rate was $58.5 \%$.

\section{Factors associated with in-hospital and 90-day mortality}

In our study, age was not found to significantly affect in-hospital or 90 -day mortality ( $\mathrm{p}=0.45$, respectively). Sex was also not significantly associated with mortality (in-hospital, $\mathrm{p}=$ 0.876; 90-day, $\mathrm{p}=0.82$ ).

Analysis showed that a lower $\mathrm{SCr}$ and blood $\mathrm{pH}$ and a higher SOFA score were independently associated with increased risk for in-hospital and 90-day mortality. A 1-SD increase in $\mathrm{SCr}$ and blood $\mathrm{pH}$ was associated with a decreased risk of in-hospital mortality (SCr: HR, 0.87; 95\% confidence interval [CI], 0.81-0.93; $\mathrm{p}<0.001$ / blood pH: HR, 0.15; 95\% CI, 0.06-0.36; $\mathrm{p}<0.001$ ) and 90-day mortality (SCr: HR, 0.84; 95\% CI, 0.79-0.90, p < 0.001 / blood pH: HR, 0.17; 95\% CI, $0.07-0.42 ; \mathrm{p}<0.001)$. On the other hand, a 1-unit increase in SOFA score was significantly associated with increased risk of in-hospital mortality (HR, 1.06; 95\% CI, 1.02- 1.09; p $<0.001$ ) and 90-day mortality (HR, 1.07; 95\% CI, 1.04-1.10; $\mathrm{p}<0.001$ ). More importantly, after adjustment for demographic factors and the other variables, $\mathrm{SCr}$, blood $\mathrm{pH}$, and SOFA score each remained significantly associated with in- 
Table 1. Baseline characteristics

\begin{tabular}{|c|c|c|c|c|}
\hline Characteristic & Total & Survivor & Non-survivor & p-value \\
\hline No. of patients & 414 & 177 & 237 & \\
\hline \multicolumn{5}{|l|}{ Demographic } \\
\hline Age (yr) & $66.4 \pm 14.8$ & $65.8 \pm 15.3$ & $66.8 \pm 14.4$ & 0.50 \\
\hline \multicolumn{5}{|l|}{ Sex } \\
\hline Male & $282(68.1)$ & $120(67.8)$ & $162(68.4)$ & 0.90 \\
\hline Female & $132(31.9)$ & $57(32.2)$ & 75 (31.6) & \\
\hline \multicolumn{5}{|l|}{ Comorbid disease } \\
\hline Hypertension & $202(48.8)$ & $99(55.9)$ & $103(43.5)$ & 0.01 \\
\hline Diabetes mellitus & $162(39.1)$ & $88(49.7)$ & $74(31.2)$ & $<0.001$ \\
\hline Malignancy & $100(24.2)$ & $28(15.8)$ & $72(30.4)$ & 0.001 \\
\hline Liver disease & $63(15.2)$ & $23(13.0)$ & $40(16.9)$ & 0.28 \\
\hline Congestive heart failure & $52(12.6)$ & $31(17.5)$ & $21(8.9)$ & 0.009 \\
\hline Myocardial infarction & $28(6.8)$ & $16(9.0)$ & $12(5.1)$ & 0.11 \\
\hline Ischemic heart disease & $21(5.1)$ & $10(5.6)$ & $11(4.6)$ & 0.64 \\
\hline COPD & $21(5.1)$ & $8(4.5)$ & $13(5.5)$ & 0.66 \\
\hline Cerebrovascular accident & $14(3.4)$ & $5(2.8)$ & $9(3.8)$ & 0.59 \\
\hline Peptic ulcer disease & 5 (1.2) & $1(0.6)$ & $4(1.7)$ & 0.30 \\
\hline MAP (mmHg) & $80.0 \pm 16.8$ & $83.2 \pm 17.6$ & $77.8 \pm 15.8$ & 0.001 \\
\hline \multicolumn{5}{|l|}{ Illness severity } \\
\hline SOFA score ${ }^{a}$ & $9.7 \pm 3.6$ & $8.9 \pm 3.2$ & $10.3 \pm .3 .8$ & $<0.001$ \\
\hline Vasopressor use & & & & $<0.001$ \\
\hline None & $119(28.7)$ & 67 (37.9) & $52(21.9)$ & \\
\hline 1 & $182(44.0)$ & $79(44.6)$ & $103(43.5)$ & \\
\hline$\geq 2$ & $113(27.3)$ & $31(17.5)$ & $82(34.6)$ & \\
\hline Use of mechanical ventilation & $276(66.7)$ & $100(56.5)$ & $176(74.3)$ & $<0.001$ \\
\hline \multicolumn{5}{|l|}{ AKI etiology ${ }^{a}$} \\
\hline Postoperative & $21(5.1)$ & $10(5.6)$ & $11(4.6)$ & 0.71 \\
\hline Septic & $285(68.8)$ & $111(62.7)$ & $174(73.4)$ & 0.004 \\
\hline Cardiogenic & $24(5.8)$ & $9(5.1)$ & $15(6.3)$ & 0.47 \\
\hline Others & $65(15.7)$ & $34(19.2)$ & 31 (13.1) & 0.11 \\
\hline Serum creatinine $(\mathrm{mg} / \mathrm{dL})^{a}$ & $3.5 \pm 2.5$ & $4.3 \pm 3.1$ & $2.9 \pm 1.7$ & $<0.001$ \\
\hline Blood $\mathrm{pH}^{\mathrm{a}}$ & $7.31 \pm 0.13$ & $7.33 \pm 0.13$ & $7.29 \pm 0.13$ & 0.006 \\
\hline UO 24 hours prior to CRRT (L) & $0.4 \pm 0.6$ & $0.4 \pm 0.5$ & $0.5 \pm 0.6$ & 0.07 \\
\hline Intensive care unit stay (day) & $9.5 \pm 14.8$ & $10.8 \pm 17.2$ & $8.5 \pm 12.7$ & 0.11 \\
\hline Hospital stay (day) & $21.2 \pm 39.3$ & $35.8 \pm 54.1$ & $10.2 \pm 15.4$ & $<0.001$ \\
\hline CRRT duration (day) & $5.1 \pm 5.6$ & $4.6 \pm 4.4$ & $5.4 \pm 6.3$ & 0.16 \\
\hline Fluid overload & $182(44.0)$ & $61(34.5)$ & $121(51.1)$ & 0.001 \\
\hline Degree of fluid overload & & & & $<0.001$ \\
\hline$\leq 10 \%$ & $115(27.8)$ & $49(27.7)$ & $66(27.8)$ & \\
\hline$>10 \%$ & $67(16.2)$ & $12(6.8)$ & $55(23.2)$ & \\
\hline
\end{tabular}

Data are presented as number only, mean \pm standard deviation for continuous variables, or number (\%) for categorical variables.

AKI, acute kidney injury; COPD, chronic obstructive pulmonary disease; CRRT, continuous renal replacement therapy; MAP, mean arterial pressure; SOFA, Sequential Organ Failure Assessment; UO, urine output.

ancomplete data. The missing data rate is $0.2 \%$ for the SOFA score, $4.6 \%$ for AKI etiology, $0.5 \%$ for serum creatinine, $1.0 \%$ for blood $\mathrm{pH}$, and $12.8 \%$ for the UO 24 hours prior to CRRT. 
hospital mortality (SCr: HR, 0.87; 95\% CI, 0.81-0.93, p $<0.001$ / blood pH: HR, 0.11; 95\% CI, 0.04-0.28; p $<0.001$ / SOFA score: HR, 1.05; 95\% CI, 1.01-1.08; $\mathrm{p}=0.008)$. By multivariate Cox regression analysis, $\mathrm{SCr}$ (HR, 0.86; 95\% CI, 0.80-0.93; p $<0.001$ ), blood pH (HR, 0.16; 95\% CI, 0.06-0.43); p < 0.001), and SOFA score (HR, 1.06; 95\% CI, 1.02-1.09; $\mathrm{p}=0.001$ ) were associated with 90-day mortality, independent of age, sex, comorbidities, and sepsis (Table 2).

FO was likewise significantly associated with an increased risk of in-hospital (HR, 1.54; 95\% CI, 1.19-1.98; $\mathrm{p}=0.001$ ) and 90-day mortality (HR, 1.57; 95\% CI, 1.22-2.02; $\mathrm{p}<0.001$ ). Patients with FO were 1.39 times (95\% CI, 1.07-1.81; $\mathrm{p}=0.02$ ) more likely to die within 90 days following CRRT initiation after adjustment for age, sex, SCr, blood pH, SOFA score, DM, hypertension, malignancy, and sepsis (Table 2).

\section{Subgroup analyses}

We also classified the patients according to degree of FO. One hundred fifteen patients (27.8\%) had FO of $\leq 10 \%$, while 67 patients $(16.2 \%)$ had more severe FO of $>10 \%$. Two hundred thirty-one patients (55.8\%) did not present with FO on CRRT initiation. Our results showed that mortality rates increased as the degree of FO increased. The highest mortality rates were recorded in patients with $\mathrm{FO}>10 \%$ (in-hospital mortality: $82.1 \%$ vs. $57.4 \%$ in FO $\leq 10 \%$, p $<0.001$; 90 -day mortality: $85.1 \%$ vs. $59.1 \%$ in $\mathrm{FO} \leq 10 \%$, p $<0.001$ ).

We further subdivided the patients into the following six groups to examine whether the increased mortality risk observed in patients with FO was adversely affected by SOFA score: group 1A, no FO and low SOFA score; group 1B, no FO and high SOFA score; group $2 \mathrm{~A}, \mathrm{FO} \leq 10 \%$ and low SOFA score; group $2 \mathrm{~B}, \mathrm{FO} \leq 10 \%$ and high SOFA score; group $3 \mathrm{~A}$, $\mathrm{FO}>10 \%$ and low SOFA score; and group $3 \mathrm{~B}, \mathrm{FO}>10 \%$ and high SOFA score (Table 3, 4).

In patients without FO (group 1), higher mortality was observed in the high SOFA subgroup (in-hospital: group 1B, $58.3 \%$ vs. group $1 \mathrm{~A}, 43.1 \%$; 90 -day: group $1 \mathrm{~B}, 58.3 \%$ vs. group 1A, 43.9\%). Hazard ratios were 1.85 (95\% CI, 1.10-3.12; $\mathrm{p}=$ 0.02 ) for in-hospital mortality (Table 3 ) and 1.79 (95\% CI, 1.06-3.02; $\mathrm{p}=0.03$ ) for 90-day mortality in group 1B (Table 4).

Among patients with FO $\leq 10 \%$, a high SOFA score (group 2B) was also associated with increased hazard ratios for both in-hospital mortality (2.92; 95\% CI, 1.53-5.59; $\mathrm{p}=0.001$ ) and 90-day mortality (3.05; 95\% CI, 1.59-5.88; $\mathrm{p}=0.001)$.

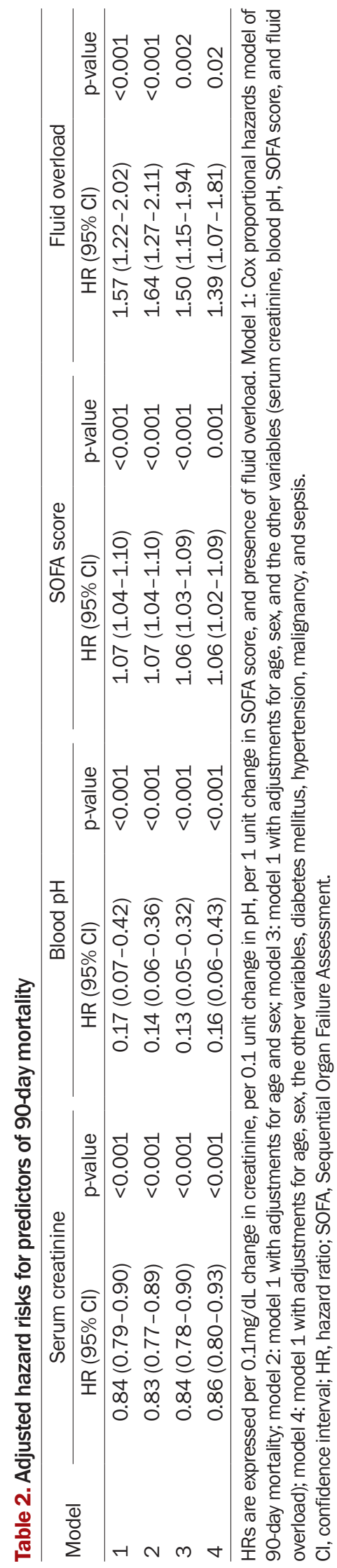


Table 3. Hazard risks of SOFA scores and fluid overload for predicting in-hospital mortality

\begin{tabular}{|c|c|c|c|c|c|c|}
\hline \multirow{2}{*}{ Fluid overload } & \multicolumn{3}{|c|}{ SOFA score $<10(n=204)$} & \multicolumn{3}{|c|}{ SOFA score $\geq 10(n=209)$} \\
\hline & No $(\%)^{a}$ & $\mathrm{HR}(95 \% \mathrm{Cl})$ & $p$-value & No $(\%)^{a}$ & $\mathrm{HR}(95 \% \mathrm{Cl})$ & p-value \\
\hline No $(n=231)$ & $53 / 70(43.1)$ & (Reference) & & $63 / 45(58.3)$ & $1.85(1.10-3.12)$ & 0.02 \\
\hline$\leq 10 \%(n=115)$ & $24 / 30(44.4)$ & $1.06(0.56-2.01)$ & 0.87 & $42 / 19(68.9)$ & $2.92(1.53-5.59)$ & 0.001 \\
\hline$>10 \%(n=67)$ & $22 / 5(81.5)$ & $5.81(2.07-16.35)$ & 0.001 & $33 / 7(82.5)$ & $6.23(2.56-15.17)$ & $<0.001$ \\
\hline
\end{tabular}

$\mathrm{Cl}$, confidence interval; HR, hazard ratio; SOFA, Sequential Organ Failure Assessment.

${ }^{a}$ Mortality/non-mortality (percentage of mortality).

Table 4. Hazard risks of SOFA scores and fluid overload for predicting 90-day mortality

\begin{tabular}{|c|c|c|c|c|c|c|}
\hline \multirow{2}{*}{ Fluid overload } & \multicolumn{3}{|c|}{ SOFA score < $10(n=204)$} & \multicolumn{3}{|c|}{ SOFA score $\geq 10(n=209)$} \\
\hline & No $(\%)^{a}$ & $\mathrm{HR}(95 \% \mathrm{Cl})$ & p-value & No $(\%)^{a}$ & $\mathrm{HR}(95 \% \mathrm{Cl})$ & $p$-value \\
\hline No $(n=231)$ & $54 / 69$ (43.9) & (Reference) & & $63 / 45(58.3)$ & $1.79(1.06-3.02)$ & 0.03 \\
\hline$\leq 10 \%(n=115)$ & $25 / 29(46.3)$ & $1.10(0.58-2.09)$ & 0.77 & $43 / 18(70.5)$ & 3.05 (1.59-5.88) & 0.001 \\
\hline$>10 \%(n=67)$ & $24 / 3$ (88.9) & $10.22(2.92-35.75)$ & $<0.001$ & $33 / 7(82.5)$ & $6.02(2.47-14.67)$ & $<0.001$ \\
\hline
\end{tabular}

$\mathrm{Cl}$, confidence interval; HR, hazard ratio; SOFA, Sequential Organ Failure Assessment.

${ }^{a}$ Mortality/non-mortality (percentage of mortality)

Among patients with a low SOFA score, it is noteworthy that the mortality risk of patients with $\mathrm{FO} \leq 10 \%$ (group $2 \mathrm{~A}$ ) was not statistically different from that of patients without FO (group 1A) (in-hospital: HR, 1.06; 95\% CI, 0.56-2.01; $\mathrm{p}=$ 0.87 / 90-day: HR, 1.10; 95\% CI, 0.58-2.09; $\mathrm{p}=0.77$ ).

Patients with FO $>10 \%$ had the highest risk of in-hospital mortality (group 3A: HR, 5.81; 95\% CI, 2.07-16.35; p = 0.001 / group 3B: HR, 6.23; 95\% CI, 2.56-15.17; p < 0.001) and 90-day mortality (group 3A: HR, 10.22; 95\% CI, 2.92-35.75; p < 0.001 / group 3B: HR, 6.02; 95\% CI, 2.47-14.67; p < 0.001) among the entire study population (Table 3,4 , respectively). The Kaplan-Meier survival curves also showed that survival was lowest in group 3 (Fig. 1, 2).

\section{Discussion}

We studied a multicenter population of 414 ICU patients with AKI who subsequently underwent CRRT. This study showed that the mortality rate was high among critically ill patients who received CRRT (in-hospital, $57.2 \%$ and 90 day, 58.5\%). Our result is comparable to mortality rates in previously published data [3,13-15] but better than those reported by Prasad et al. [16] (64\%), Kao et al. [17] (66.5\%), and Gonzalez et al. [18] (68.4\%). Some studies have even reported in-hospital mortality as high as $70 \%-80 \%[19,20]$.

Several articles have previously cited male sex, older age, and sepsis as risk factors for severe AKI requiring RRT [3,8]. Similar to previous studies, a majority of the patients in this study were male $(282,68.1 \%)[17,21]$. The mean age was 66.4 \pm 14.8 years, and most of the patients were $\geq 65$ years ( 250 , $60.4 \%)$. The most common etiology of AKI was sepsis, as was the case in the studies conducted by Kao et al. [17], Gonzalez et al. [18], and Soni et al. [19] .

The association between advanced age and mortality among AKI patients has been extensively studied [17-19]. In a study by Allegretti et al. [14] of 725 AKI patients who received CRRT, age over 60 years was an independent risk factor of in-hospital mortality (odds ratio [OR], 1.9; 95\% CI, 1.3-2.7; $\mathrm{p}=0.001$ ) and mortality following hospital discharge (HR, 1.9; 95\% CI, 1.2-3.0). Another study by Conroy et al. [22] reported that patients 75 years and older had higher hospital mortality ( $54.2 \%$ vs. $44 \%, \mathrm{p}=0.02$ ) and 1-year mortality (63.6\% vs. $50.6 \%, \mathrm{p}=0.005)$ than younger patients. Although poor outcomes have been observed in elderly AKI patients, this has not been consistent across all studies [23]. A retrospective study done in Germany of 424 patients found that the course and prognosis of AKI do not differ greatly in the elderly population [24]. Our study also did not identify age as a predictor of mortality in patients with CRRT-requiring AKI.

A lower SCr and blood $\mathrm{pH}$ and a higher SOFA score were independently associated with an increased risk of death. Several studies have identified high $\mathrm{SCr}$ as an independent factor for better outcomes [20,25]. Soubrier et al. [26], in a study of 197 patients treated with CRRT, found that SCr > $3.4 \mathrm{mg} / \mathrm{dL}$ predicted a favorable outcome. In our study, the non-survivors had a lower mean SCr at CRRT initiation. 


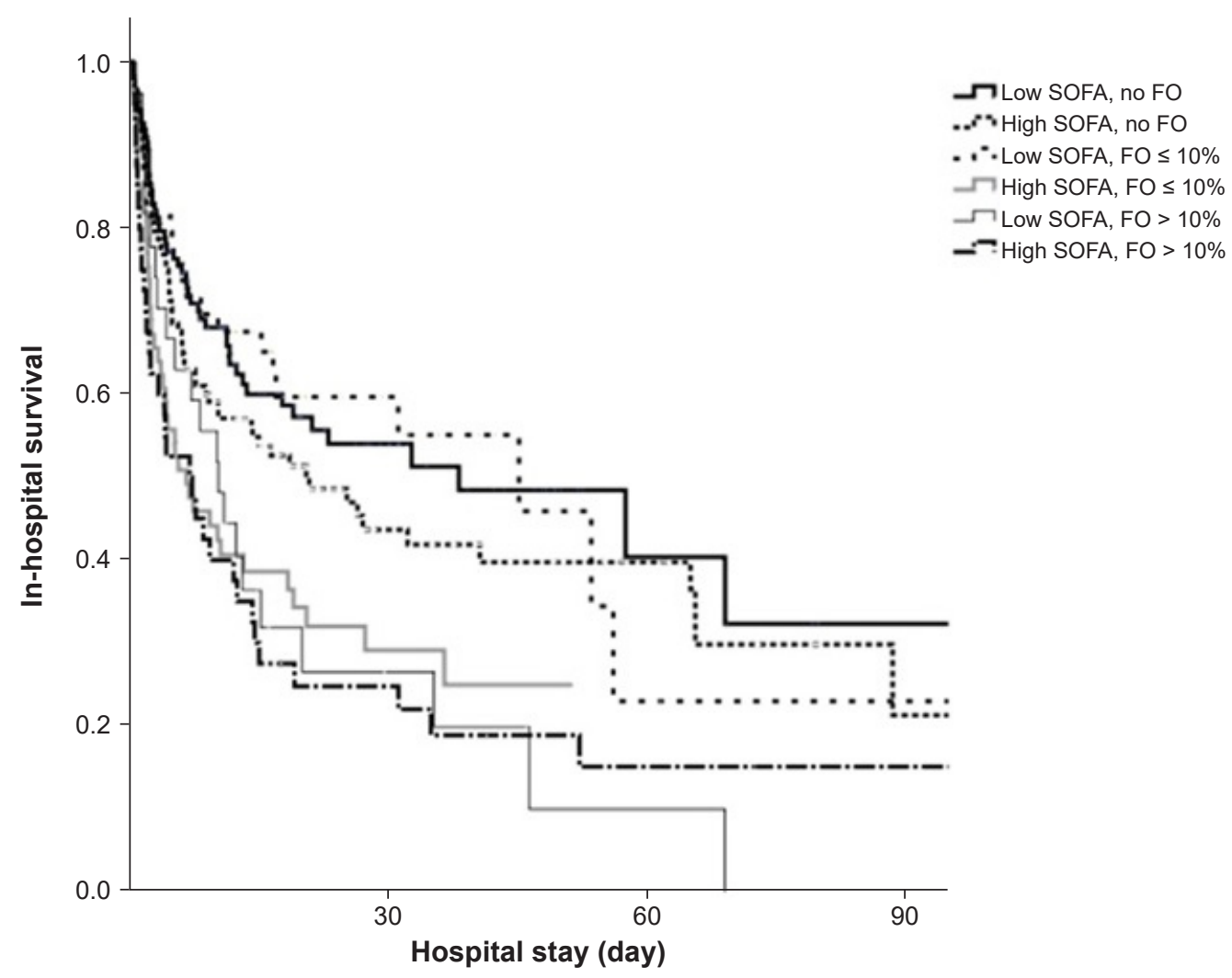

\begin{tabular}{lrrrr} 
Patients-at-risk & & & & \\
Low SOFA, no FO & 123 & 27 & 5 & 1 \\
High SOFA, no FO & 108 & 24 & 14 & 5 \\
Low SOFA, FO $\leq 10 \%$ & 54 & 14 & 2 & 1 \\
High SOFA, FO $\leq 10 \%$ & 61 & 9 & 0 & 0 \\
Low SOFA, FO > 10\% & 27 & 4 & 1 & 0 \\
High SOFA, FO > 10\% & 40 & 9 & 4 & 2 \\
\hline Total & 413 & 78 & 26 & 9
\end{tabular}

Figure 1. Kaplan-Meier curves of in-hospital survival among the six groups.

FO, fluid overload; SOFA, Sequential Organ Failure Assessment.

A 1-SD increase was associated with a decreased risk of in-hospital and 90-day mortality (HR, 0.87 and 0.84 , respectively; $\mathrm{p}<0.001$ ). Possible explanations for this occurrence are decreased protein production and reduced muscle mass among the more critically ill patients. SOFA represents a severity parameter and is widely accepted as a prognostic factor for critically ill patients. In a study of 240 patients with AKI who received CRRT, Kee et al. [27] found that blood $\mathrm{pH}$ $<7.35$ (OR, 4.33; 95\% CI, 2.41-7.77; $\mathrm{p}<0.001$ ) and a 1-SD increase in SOFA score (OR, 1.99; 95\% CI, 1.49-2.69; p < 0.001) were significantly associated with mortality within 7 days of CRRT initiation. Another single-center, retrospective cohort study of 562 patients also reported that acidemia and a higher SOFA score at the time of CRRT initiation were independently associated with a higher short-term mortality rate (death in-hospital or within one week of discharge) [28].

FO is known to be associated with mortality in critically ill patients with AKI $[4,25,29]$. Similar to other studies, and not surprisingly, FO was associated with increased mortality in our study. Although most studies have defined FO as more than a $10 \%$ increase in body weight relative to baseline, we included patients with less than $10 \%$ increase in body weight 


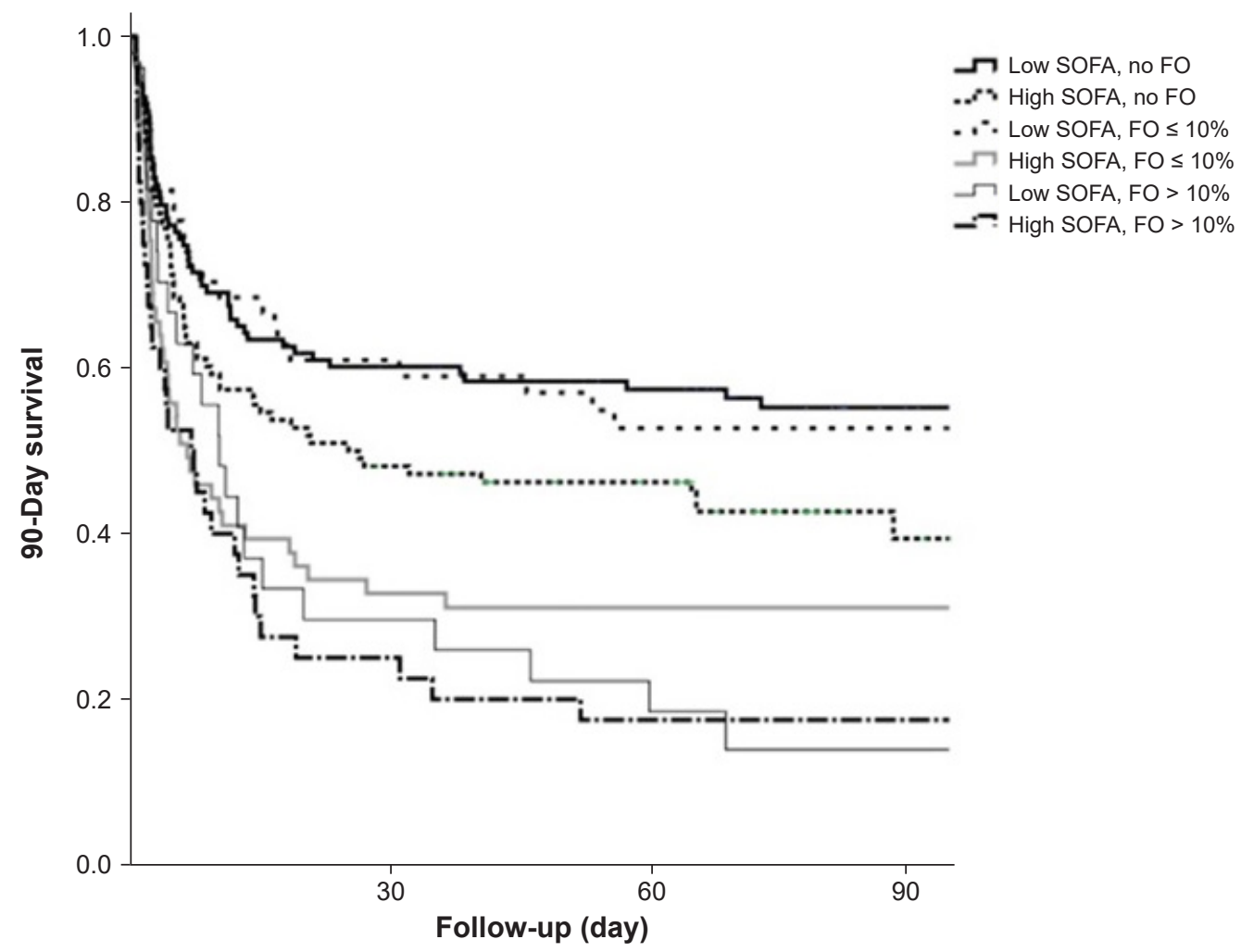

\begin{tabular}{lllll} 
Patients-at-risk & & & & \\
Low SOFA, no FO & 123 & 72 & 59 & 40 \\
High SOFA, no FO & 108 & 51 & 43 & 24 \\
Low SOFA, FO $\leq 10 \%$ & 54 & 31 & 24 & 18 \\
High SOFA, FO $\leq 10 \%$ & 61 & 20 & 15 & 14 \\
Low SOFA, FO > 10\% & 27 & 8 & 7 & 3 \\
High SOFA, FO > 10\% & 40 & 10 & 7 & 7 \\
\hline Total & 413 & 192 & 155 & 106
\end{tabular}

Figure 2. Kaplan-Meier curves of 90-day survival among the six groups.

FO, fluid overload; SOFA, Sequential Organ Failure Assessment.

from baseline in our analysis since a study by Bagshaw et al. [30] found that a lower threshold of fluid accumulation (>5\%) was associated with hospital mortality (OR, 2.31). This negative effect of FO $\leq 10 \%$ on survival was more clearly seen in patients presenting with more severe illness (group 2B). Patients with a lesser degree of FO $(<10 \%)$ should be given more attention since they are also at an increased risk for adverse outcomes. Timely recognition and subsequent management of FO in its earlier stages could positively impact patients' hospital course and long-term prognosis.

Moreover, our results showed that a higher degree of FO was associated with higher risk of mortality. We observed a relationship between increasing degree of FO, increasing SOFA score, and mortality. In a study of 341 AKI patients who underwent CRRT, Kim et al. [31] observed that the adverse effect of $\mathrm{FO}$ on survival was more evident in patients with sepsis or more severe illness. In contrast, our results revealed that the increased risk of mortality associated with FO was also observed in patients with a low SOFA score. Patients with FO $>10 \%$ and low SOFA score (group 3A) were 5.8 times more likely to die in-hospital than patients with no FO and low SOFA score (group 1A). This risk increased to 6.3 when patients had high SOFA score and FO > 10\% (group 3B). This highlights the importance of proper fluid manage- 
ment among critically ill patients with AKI regardless of disease severity.

This study showed that mortality following CRRT initiation for AKI was high. Our study demonstrated that SCr, blood $\mathrm{pH}$, SOFA score, and FO are significant independent risk factors for in-hospital and 90-day mortality after adjustment for age, sex, sepsis, and comorbidities. In patients without FO and with FO $\leq 10 \%$, a lower SOFA score corresponded to a lower risk of in-hospital and 90-day mortality. Patients with FO $>10 \%$ had worse outcomes regardless of SOFA score.

In conclusion, the presence of FO signifies an increased risk of mortality independent of other factors, including severity of acute illness. Prevention of FO should be a priority, especially when managing the critically ill. Measures to ensure this include correctly identifying patients who are fluid-responsive, choosing the appropriate type and quantity of fluids to be given, and frequent clinical assessment of fluid status. Benefits and risks should always be weighed prior to starting or deciding to continue fluid therapy. A judicious fluid therapy is indispensable in the management of critically ill patients.

Although the predictors we identified in this study were identified separately in previous studies, our findings reaffirm the clinical importance of these factors in the management and prognosis of critically ill patients with AKI. This study has several strengths. It is a multicenter study and also included a larger number of patients compared to previous studies. Our findings can be extended to other ICU patients. This is an observational study. Interventions were not standardized. The decision to start CRRT, choice of CRRT modality, and CRRT prescription were made by the attending nephrologist. Our results can only predict the associations between factors and outcomes but do not determine causal relationship.

\section{Conflicts of interest}

All authors have no conflicts of interest to declare.

\section{Funding}

This research was supported by the National Research Foundation (grant No. 2019R1A2C1085411) and the Korea Health Technology R\&D Project through the Korea Health Industry Development Institute (KHIDI), funded by the Ministry of
Health \& Welfare, Republic of Korea (grant No. HI17C1827).

\section{Authors' contributions}

Conceptualization, Methodology: All authors

Data curation: KPML, JCJ, SK

Writing-original draft: KPML

Writing-review \& editing: JCJ, SK

All authors read and approved the final manuscript.

\section{ORCID}

Kristianne Rachel P. Medina-Liabres, https://orcid.org/00000003-1092-1930

Jong Cheol Jeong, https://orcid.org/0000-0003-0301-7644

Hyung Jung Oh, https://orcid.org/0000-0002-4281-696X

Jung Nam An, https://orcid.org/0000-0001-5108-1005

Jung Pyo Lee, https://orcid.org/0000-0002-4714-1260

Dong Ki Kim, https://orcid.org/0000-0002-5195-7852

Dong-Ryeol Ryu, https://orcid.org/0000-0002-5309-7606

Sejoong Kim, https://orcid.org/0000-0002-7238-9962

\section{References}

1. Kellum JA, Angus DC, Johnson JP, et al. Continuous versus intermittent renal replacement therapy: a meta-analysis. Intensive Care Med 2002;28:29-37.

2. Tolwani A. Continuous renal-replacement therapy for acute kidney injury. N Engl J Med 2012;367:2505-2514.

3. De Corte W, Dhondt A, Vanholder R, et al. Long-term outcome in ICU patients with acute kidney injury treated with renal replacement therapy: a prospective cohort study. Crit Care 2016;20:256.

4. Vaara ST, Korhonen AM, Kaukonen KM, et al. Fluid overload is associated with an increased risk for 90-day mortality in critically ill patients with renal replacement therapy: data from the prospective FINNAKI study. Crit Care 2012;16:R197.

5. Tandukar S, Palevsky PM. Continuous renal replacement therapy: who, when, why, and how. Chest 2019;155:626-638.

6. Friedericksen DV, Van der Merwe L, Hattingh TL, Nel DG, Moosa MR. Acute renal failure in the medical ICU still predictive of high mortality. S Afr Med J 2009;99:873-875.

7. Acheampong A, Vincent JL. A positive fluid balance is an independent prognostic factor in patients with sepsis. Crit Care 2015;19:251. 
8. Payen D, de Pont AC, Sakr Y, et al. A positive fluid balance is associated with a worse outcome in patients with acute renal failure. Crit Care 2008;12:R74.

9. Sirvent JM, Ferri C, Baró A, Murcia C, Lorencio C. Fluid balance in sepsis and septic shock as a determining factor of mortality. Am J Emerg Med 2015;33:186-189.

10. Han MJ, Park KH, Shin JH, Kim SH. Influence of daily fluid balance prior to continuous renal replacement therapy on outcomes in critically ill patients. J Korean Med Sci 2016;31:13371344.

11. Khwaja A. KDIGO clinical practice guidelines for acute kidney injury. Nephron Clin Pract 2012;120:c179-c184.

12. Gemmell L, Docking R, Black E. Renal replacement therapy in critical care. BJA Educ 2017;17:88-93.

13. Park JY, An JN, Jhee JH, et al. Early initiation of continuous renal replacement therapy improves survival of elderly patients with acute kidney injury: a multicenter prospective cohort study. Crit Care 2016;20:260.

14. Allegretti AS, Steele DJ, David-Kasdan JA, Bajwa E, Niles JL, Bhan I. Continuous renal replacement therapy outcomes in acute kidney injury and end-stage renal disease: a cohort study. Crit Care 2013;17:R109.

15. Wu L, Zhang P, Yang Y, et al. Long-term renal and overall survival of critically ill patients with acute renal injury who received continuous renal replacement therapy. Ren Fail 2017;39:736-744.

16. Prasad B, Urbanski M, Ferguson TW, Karreman E, Tangri N. Early mortality on continuous renal replacement therapy (CRRT): the prairie CRRT study. Can J Kidney Health Dis 2016;3:36.

17. Kao CC, Yang JY, Chen L, et al. Factors associated with poor outcomes of continuous renal replacement therapy. PLoS One 2017;12:e0177759.

18. Gonzalez CA, Pinto JL, Orozco V, et al. Early mortality risk factors at the beginning of continuous renal replacement therapy for acute kidney injury. Cogent Med 2018;5:1407485.

19. Soni SS, Nagarik AP, Adikey GK, Raman A. Using continuous renal replacement therapy to manage patients of shock and acute renal failure. J Emerg Trauma Shock 2009;2:19-22.

20. Aldawood A. Outcome and prognostic factors of critically ill patients with acute renal failure requiring continuous renal replacement therapy. Saudi J Kidney Dis Transpl 2010;21:11061110.

21. Uchino S, Bellomo R, Morimatsu H, et al. Continuous renal replacement therapy: a worldwide practice survey: the beginning and ending supportive therapy for the kidney (B.E.S.T. kidney) investigators. Intensive Care Med 2007;33:1563- 1570.

22. Conroy M, O’Flynn J, Marsh B. Mortality and long-term dialysis requirement among elderly continuous renal replacement therapy patients in a tertiary referral intensive care unit. J Intensive Care Soc 2019;20:138-143.

23. Medina-Liabres KR, Kim S. Continuous renal replacement therapy in elderly with acute kidney injury. Korean J Intern Med 2020;35:284-294.

24. Funk I, Seibert E, Markau S, Girndt M. Clinical course of acute kidney injury in elderly individuals above 80 years. Kidney Blood Press Res 2016;41:947-955.

25. Gleeson PJ, Crippa IA, Sexton DJ, et al. Determinants of renal recovery and mortality inpatients undergoing continuous renal replacement therapy in the ICU. Intensive Care Med Exp 2015;3:A54.

26. Soubrier S, Leroy O, Devos P, et al. Epidemiology and prognostic factors of critically ill patients treated with hemodiafiltration. J Crit Care 2006;21:66-72.

27. Kee YK, Kim D, Kim SJ, et al. Factors associated with early mortality in critically ill patients following the initiation of continuous renal replacement therapy. J Clin Med 2018;7:334.

28. Rhee H, Jang KS, Park JM, et al. Short- and long-term mortality rates of elderly acute kidney injury patients who underwent continuous renal replacement therapy. PLoS One 2016;11:e0167067.

29. Bouchard J, Soroko SB, Chertow GM, et al. Fluid accumulation, survival and recovery of kidney function in critically ill patients with acute kidney injury. Kidney Int 2009;76:422-427.

30. Bagshaw SM, Chakravarthi MR, Ricci Z, et al. Precision continuous renal replacement therapy and solute control. Blood Purif 2016;42:238-247.

31. Kim IY, Kim JH, Lee DW, et al. Fluid overload and survival in critically ill patients with acute kidney injury receiving continuous renal replacement therapy. PLoS One 2017;12:e172137. 